\title{
La "ambientalización" de los estudios de ciencias ambientales en España
}

\author{
The "greening" of environmental sciences \\ studies in Spain
}

\section{Ana Isabel Luque Palmero Francisco Javier Perales Palacios}

Dpto. de Didáctica de las Ciencias Experimentales Universidad de Granada

Resumen: Presentamos los resultados de un proceso de identificación de la presencia de la Educación Ambiental (EA) en los grados de Ciencias Ambientales (CA) en España. Para ello hemos indagado en las páginas web de las universidades, señalando la presencia de la EA, su carácter optativo/obligatorio, los departamentos responsables de la misma y los indicadores de EA y de metodología docente en sus guías docentes. Por otro lado se aplicó una encuesta a una muestra de estudiantes universitarios del grado de CA sobre su interés hacia la EA, la naturaleza de esta y su grado de acuerdo con los indicadores anteriores. Los resultados evidencian una implantación parcial de la EA y una esperanzadora actitud de los estudiantes hacia dicha presencia.

Palabras clave: Educación Ambiental, Educación para la Sostenibilidad, Grado de Ciencias Ambientales, Ambientalización, Profesionalización de la Educación Ambiental.

Abstract: We show the results of a process of identifying the presence of Environmental Education (EE) in Environmental Sciences degrees (ES) in Spain. So we have searched into the websites of universities, pointing the presence of $\mathrm{EE}$, the optional/compulsory character, the departments responsible for it, and indicators of EE and teaching methodology in their teaching guides. On the other hand a survey was applied to a sample of students of university degree of ES including their interest in the EE, the nature of this, and how much they agree with the above indicators. The results show a partial implementation of the EE and a hopeful attitude of students towards such presence.

Keywords: Environmental Education, Education for Sustainability, Environmental Sciences Degree, Greening, Professionalization of Environmental Education.

(Fecha de recepción: julio, 2015, y de aceptación: marzo, 2016)

DOI: 10.7203/DCES.30.6444 


\section{Introducción}

Como es bien conocido, hace escasos años ha concluido en España la progresiva implementación de los nuevos grados universitarios acogidos al Espacio Europeo de Educación Superior. Ello ha supuesto una profunda renovación de las titulaciones para ajustarse a las directrices del proceso de Bolonia, recientemente cuestionado en cuanto a la duración de los estudios de grado y posgrado por el actual gobierno (Real Decreto 43/2015, de 2 de febrero). Una de las premisas del nuevo marco curricular ha sido la deseable promoción de las competencias profesionales entre los futuros graduados, lo que ha obligado a los diseñadores de los nuevos planes a contemplar en sus memorias de verificación los distintos itinerarios profesionales y, en algunos casos, a dar satisfacción a las mismas en su oferta académica.

En este artículo nos hemos planteado la pregunta de si la afirmación anterior es válida para la presencia de la Educación Ambiental (EA, en adelante) en los nuevos grados de Ciencias Ambientales (CA, en adelante). De aquí que en el título de este artículo hayamos incluido el término "ambientalización" (García, 2004) para referirnos a tal presencia, aunque somos conscientes de que dicho término debería ser entendido, en un sentido más genérico, como impregnación de actitudes y valores proambientales en los planes de estudios de dicho grado, lo que entroncaría con la consideración de la EA como "área transversal" introducida por la LOGSE (Ley Orgánica 1/1990, de 3 de Octubre).
Como es bien sabido, la EA (o Educación para la Sostenibilidad, como alternativamente es denominada) comenzó a configurarse como materia de estudio a partir de la década de los 70 del siglo pasado, emanada de las primeras advertencias sobre lo finito de nuestros recursos naturales y la necesidad de limitar nuestro impacto sobre el medio natural.

Así, ya la UICN en el año 1970 definía a la EA como: "el proceso que consiste en reconocer valores y aclarar conceptos con objeto de fomentar las aptitudes y actitudes necesarias para comprender y apreciar las interrelaciones entre el hombre, su cultura y su medio biofísico. Entraría también la práctica en la toma de decisiones y en la propia elaboración de un código de comportamiento con respecto a las cuestiones relacionadas con la calidad del Medio Ambiente" (Carta de Nevada, UICN, 1970). Características que aún hoy día mantienen su vigencia.

Uno de los grandes retos de la EA ha sido el de formar profesionales cualificados que pudieran afrontar los desafíos que nuestro mundo tiene planteados y que pudieran convertirse, a su vez, en formadores de formadores. Hay que tener en cuenta que, salvo honrosas excepciones, la EA adolece de presencia significativa en el contexto educativo formal y en la práctica docente habitual, por lo que para la mayoría de los ciudadanos la única formación ambiental de que disponen la han adquirido por los medios de comunicación, con las limitaciones que ello conlleva (Perales, 2010a). 
En nuestro país solo recientemente se ha ofertado el título de Monitor de Educación Ambiental dentro del Programa de Formación Profesional Ocupacional del Ministerio de Trabajo y Asuntos Sociales, con una duración total de 300 horas $^{1}$.

$\mathrm{Al}$ margen de esa escasez de oferta educativa, el empleo verde en el campo de la Educación e Información Ambiental ha pasado de 900 empleos en el año 1998 a 7.871 en el 2009 (Fundación Biodiversidad, 2010, p. 60), ello a pesar de los obstáculos que limitan su deseable expansión extraídos de un amplio estu- dio sobre empresas españolas del sector ambiental (Tabla I).

El objetivo básico de este trabajo ha sido doble: (1) describir y analizar la oferta académica en EA que incluyen los nuevos grados de CA en España y (2) complementariamente, recabar información sobre la percepción que una muestra de estudiantes de dicho grado de la Universidad de Granada posee acerca de la naturaleza, importancia y necesidad de la EA. Este último podría validar (o no) la presencia de la EA en sus estudios universitarios.

\title{
Tabla I
}

\section{Factores actuales que impiden la expansión del sector de información y EA}

\author{
(Fuente: Fundación Biodiversidad ${ }^{2}, 2010$, p. 214)
}

\begin{tabular}{|l|c|}
\hline \multicolumn{1}{|c|}{ Obstáculos } & Porcentaje en el que afectan \\
\hline Crisis económica & 63,3 \\
\hline $\begin{array}{l}\text { Débil conciencia y sensibilidad ambiental por parte de los ciuda- } \\
\text { danos y de la industria }\end{array}$ & 40,3 \\
\hline $\begin{array}{l}\text { Elevada inversión inicial que suponen para la empresa las actua- } \\
\text { ciones ambientales }\end{array}$ & 28,8 \\
\hline $\begin{array}{l}\text { No consideración por parte de las empresas de la actuación } \\
\text { ambiental como factor de competitividad }\end{array}$ & 21,1 \\
\hline Aversión al cambio por parte de las estructuras empresariales & 20,3 \\
\hline $\begin{array}{l}\text { Bajo grado de cumplimiento de la legislación ambiental y poco } \\
\text { control ejercido por parte de la administración }\end{array}$ & 16,9 \\
\hline $\begin{array}{l}\text { Dificultad por parte de las empresas para adaptarse a la legisla- } \\
\text { ción ambiental. }\end{array}$ & 15,3 \\
\hline Legislación ambiental poco exigente & 11,8 \\
\hline Otros factores & 11,5 \\
\hline Bajo nivel de desarrollo de la tecnología & 9,4 \\
\hline Ns / Nc & 15,4 \\
\hline
\end{tabular}

\footnotetext{
${ }^{1} \mathrm{http} / / /$ formacionavicena.com/documentos/SS-CS20.pdf.

$2 \mathrm{http} / / /$ www.forumambiental.org/pdf/Empleo_Verde.pdf.
} 
Tal objetivo requiere partir de unos fundamentos teóricos que poseen relación con la percepción que la población (estudiantes de Ciencias Ambientales en nuestro caso) posee sobre los problemas ambientales y sus soluciones, así como de la Evaluación de Programas.

En un trabajo anterior (Perales, 2010b) hemos indagado en la percepción de la situación ambiental por parte de estudiantes de Maestro de Educación Primaria y sus variaciones temporales, evidenciando una gran estabilidad de aquella. Básicamente nuestros estudiantes están sensibilizados ante los innumerables problemas ambientales que nos afectan y son conscientes de la responsabilidad que la sociedad, las instituciones y los individuos poseen en ello. A este respecto imputan fundamentalmente este estado de cosas al consumo excesivo y reclaman la necesidad del reciclaje, de una toma de conciencia generalizada y de un régimen sancionador severo para poder comenzar a cambiar. No obstante, cuando indagamos en los posibles cambios producidos por la asignatura de Educación Medioambiental sobre sus actitudes y comportamientos ambientales, estos también muestran una gran persistencia. Ese hecho quizás pudiera hacernos pensar que el paradigma cultural de nuestra sociedad (Tábara, 2001) condiciona en gran medida el pensamiento y la acción de los jóvenes.

Por lo que respecta a los modelos de evaluación de los programas educativos, estos pueden ser clasificados básicamente en dos tipos: los experimenta- les y los semicualitativos o cualitativos (Medrano, 1997), teniendo los primeros una naturaleza más basada en el producto de la intervención educativa y los segundos una más basada en el proceso de intervención. Según este último autor, los criterios en los que se puede basar la evaluación pueden agruparse en (Medrano, 1997, p. 105):

1) Aspectos generales del proyecto, planteamientos globales, destinatarios, etc.

2) Programa Educativo:objetivos, metodología y contenidos.

3) Los recursos utilizados: documentos y materiales de apoyo.

4) Personal responsable de llevar a cabo el programa.

5) Características de los equipamientos donde se desarrolla el programa.

6) La Evaluación del Programa.

En este trabajo nos fijaremos en los dos primeros criterios para la evaluación que llevaremos a cabo.

En cuanto a los programas de EA, como señalan Carleton-Hug y Hug (2010), existe un considerable déficit en la cultura de evaluación de los mismos, sugiriendo varios ámbitos en los que podrían centrarse y las oportunidades que ello conllevaría.

Para Athman y Monroe (2001) los programas de EA efectivos deberían ser relevantes para el organismo promotor, para los objetivos educativos de los receptores del mismo y para las vidas diarias de estos, habiendo de implicar a los diseñadores en todas sus 
fases de desarrollo y evolución, empoderando a los aprendices con recursos para afrontar los retos con sentido de la responsabilidad e incorporar múltiples perspectivas con un sentido interdisciplinar, empleando las mejores prácticas educativas y ser evaluados con los instrumentos adecuados.

A continuación vamos a exponer, en primer lugar, la metodología seguida en el trabajo y, posteriormente, iremos describiendo y discutiendo los datos obtenidos, tanto relativos a la presencia de la EA en los distintos planes de estudio como a la encuesta realizada, concluyendo con unas reflexiones finales.

\section{Metodología}

Al tratarse de un estudio descriptivo, la metodología ha tenido un carácter mixto cualitativo-cuantitativo. Como hemos anticipado, dicho estudio podría enmarcarse en la tipología de evaluación de programas, existiendo escasos precedentes similares en el ámbito español (Sureda et al., 2014), así como en el de la percepción ambiental (Gil, 2004).

La búsqueda de información sobre la presencia de la EA en los planes de estudio de CA, de sus memorias de verificación y de sus guías docentes se efectuó a través de la consulta de las páginas web oficiales de todas las universidades españolas. En concreto, el análisis documental se centró en el carácter optativo/obligatorio de la materia de EA, su adscripción a departamentos y áreas de conocimiento, así como en la presencia de un itinerario profesional de EA (en las memorias de verificación) y de las guías docentes, disgregándose las competencias previstas la metodología de enseñanza prevista. Para disponer de una perspectiva temporal, comenzaremos por mostrar los antecedentes de la presencia de la EA en los anteriores planes de estudio (licenciaturas en CA).

En cuanto a la encuesta pasada a los alumnos (ver más adelante las Tablas VI y VI), combinó preguntas tipo-Likert con preguntas abiertas, teniendo un carácter anónimo a fin de favorecer la veracidad de las respuestas. Las cuestiones planteadas versaban sobre su interés hacia la EA así como sobre su opinión acerca de las características de la misma a partir de diversos indicadores extraídos de la literatura educativa. Este tipo de encuestas se ha venido utilizando en otros ámbitos, sean de la educación formal o informal (p. ej., Gil, 2004; Perales, 2006; CIS, 2007; $\mathrm{CE}, 2014$ ), incluyendo las competencias que se tratan de desarrollar (Ponce y Tójar, 2014). A juicio de Tábara (2001, p. 130), en los estudios sobre percepción ambiental se debería tratar de medir:

a) El estudio del nivel de preocupación y la estimación de la gravedad o importancia de los diferentes problemas y riesgos ambientales.

b) El examen del grado de información y de conocimiento de la problemática ambiental; las fuentes de información más utilizadas; y la evaluación pública de tales fuentes.

c) La exploración de los comportamientos ambientales; el apoyo ciudadano 
a la protección del medio ambiente y las preferencias y disponibilidades de la población a llevar a cabo o a aceptar, medidas políticas de carácter ambiental.

d) El análisis de contrastes que aparecen en los anteriores temas en función de las diferencias observables en las características socioeconómicas de los individuos.

En nuestro caso, la encuesta utilizada, de acuerdo al objetivo de la investigación, puede ubicarse en el apartado c).

Participó una muestra de 87 estudiantes del grado de CA de la Universidad de Granada, escogidos aleatoriamente, por lo que procedían de diversos cursos.

El análisis de datos consistió en el recuento de frecuencias y porcentajes correspondientes para la presencia de la EA en los nuevos grados, junto con la categorización inductiva-deductiva para las respuestas a las cuestiones abiertas presentes en la encuesta y para el análisis de contenido de las guías docentes.

\section{Resultados. Situación de la Educación Ambiental en los estudios de Ciencias Ambientales}

En los apartados 3.1 y 3.2 abordaremos el objetivo (1) y en el 3.3 el (2).

\section{Antecedentes}

Comenzaremos por referirnos a la presencia de la EA en los títulos ya extinguidos de CA. Para ello llevamos a cabo una revisión de la información presente en las páginas web de las universidades españolas en el año 2012, arrojando que, en primer lugar, eran un $40 \%$ de ellas las que incorporaban los estudios de CA y, de éstas, un $43 \%$ incluían entre su oferta docente a la EA, teniendo en todos los casos carácter de asignatura optativa y oscilando su duración entre 4,5 y 6 créditos.

\section{La EA en el grado de CA}

Presencia de la EA en los nuevos grados en CA

Si nos fijamos ahora en el nuevo grado de CA, mostramos en la Tabla II el listado de las universidades que ofertan esa titulación y las que lo hacen con la EA en su plan de estudios, incluyendo asimismo su denominación. El criterio de selección ha sido que el título de las asignaturas incluyera el término de EA.

El porcentaje de universidades que contemplan la asignatura EA en su plan de estudios en los títulos de grado es superior al que había en la licenciatura, con un $65 \%$ del total (Figura I). Por otra parte, frente a un $100 \%$ de carácter optativo en las antiguas licenciaturas se ha pasado a un 55\% de optatividad y el consiguiente $45 \%$ de obligatoriedad. La duración de tales asignaturas oscila en una horquilla de 3-6 créditos. 


\section{Tabla II}

Universidades españolas que ofertan (o no) EA en sus grados de CA y denominación de las asignaturas

\begin{tabular}{|c|c|c|}
\hline $\begin{array}{c}\text { Universidades que ofertan } \\
\text { el Grado de CA }\end{array}$ & $\begin{array}{c}\text { Grados que } \\
\text { incluyen la EA }\end{array}$ & Denominación de la materia \\
\hline Alcalá & Sí & EA \\
\hline Alfonso X el Sabio & No & \\
\hline Almería & No & \\
\hline Autónoma de Barcelona & Sí & Comunicación y EA \\
\hline Autónoma de Madrid & Sí & Interpretación y EA \\
\hline Cádiz & No & \\
\hline Barcelona & No & \\
\hline Católica Santa Teresa de Jesús de Ávila & Sí & Comunicación y EA \\
\hline Castilla la Mancha & Sí & EA \\
\hline Córdoba & Sí & EA \\
\hline Europea Miguel de Cervantes & Sí & EA \\
\hline Extremadura & No & \\
\hline Girona & No & \\
\hline Granada & Sí & Formación y EA \\
\hline Huelva & Sí & EA \\
\hline Jaén & Sí & EA \\
\hline León & Sí & $\begin{array}{l}\text { Educación y Comunicación } \\
\text { Ambiental }\end{array}$ \\
\hline Miguel Hernández de Elche & Sí & EA \\
\hline Murcia & Sí & EA \\
\hline Nacional de Educación a Distancia & Sí & EA \\
\hline Pablo de Olavide & Sí & Intervención Social y EA \\
\hline País Vasco & Sí & EA y prácticas sociales \\
\hline Politécnica de Cataluña & No & \\
\hline Politécnica de Valencia & Sí & EA \\
\hline Rey Juan Carlos & No & \\
\hline Salamanca & Sí & EA \\
\hline Valencia & Sí & Interpretación y EA \\
\hline Vic & No & \\
\hline Vigo & No & \\
\hline Zaragoza & Sí & EA \\
\hline
\end{tabular}


Este hecho podría interpretarse como una evolución positiva respecto de la presencia de la asignatura en los títulos de CA, especialmente si se tiene en cuenta que se ha pasado de una carga global de 300 créditos de las licenciaturas a una de 240 en los grados.

\section{Figura I}

Universidades españolas que ofertan la asignatura de EA en el grado de CA

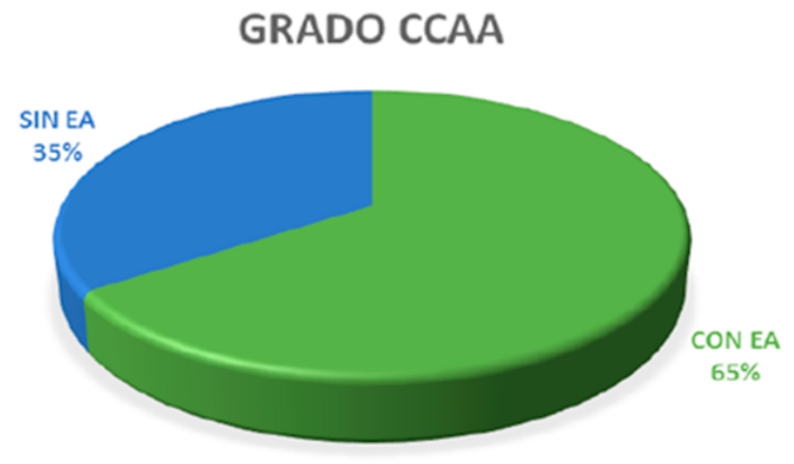

La EA en las memorias de verificación de los grados

Revisando las memorias de verificación de los grados en CA, hemos constatado que se menciona a la EA como salida profesional en 28 de las 29 universidades que se han analizado, a pesar de que un $35 \%$ de ellas no incluye siquiera una asignatura relacionada con el ámbito correspondiente a la EA.

En este último caso, cabe suponer que la mención a la EA en las salidas profesionales puede deberse, por ejemplo, a que se acepta la relevancia de la misma en el ámbito laboral, pero también podría ser que se incluyera solo para engrosar el itinerario laboral, ya que luego el plan de estudios no se corresponde con esa perspectiva.

A continuación vamos a referirnos a los departamentos a los que se vincula la EA. El resultado obtenido es de 17 departamentos distintos contabilizando las 20 universidades que la ofertan. Sin embargo, en una segunda aproximación y para hacer más sencilla la idea que se pretende mostrar aquí, se han agrupado todos los departamentos en cuatro subgrupos según la similitud de las áreas de conocimiento de cada departamento (Tabla III).

Como cabe apreciar, el mayor porcentaje corresponde a departamentos relacionados con la biología, la ecología o la botánica, seguido estrechamente por los departamentos dedicados a la didáctica. Es interesante resaltar que para una misma asignatura exista esa diversidad de departamentos responsables, lo que permite presumir también orientaciones claramente diferenciadas en la forma de entender la EA. A 


\section{Tabla III}

\section{Adscripción de la EA a grupos de departamentos universitarios}

\begin{tabular}{|c|c|}
\hline Grupo departamental & Porcentaje \\
\hline Biología/Botánica/Ecología & $42 \%$ \\
\hline Educación/Didáctica & $32 \%$ \\
\hline Ciencias Sociales/Sociología & $16 \%$ \\
\hline Economía & $10 \%$ \\
\hline
\end{tabular}

este respecto parece existir una clara división entre "ciencias" y "letras" sin sinergias entre ambos grupos. Solo los departamentos de didácticas de las ciencias experimentales o sociales podrían salvar esa frontera por cuanto el profesorado posee una doble formación científico-didáctica.
A continuación, se representa un gráfico con la distribución de créditos de la asignatura de EA según su porcentaje de aparición (Figura II).

Como puede comprobarse, predominan las materias con seis créditos sobre el resto.

Figura II

Número de créditos de las asignaturas de EA agrupados en porcentajes

\section{\% n CRÉDITOS}

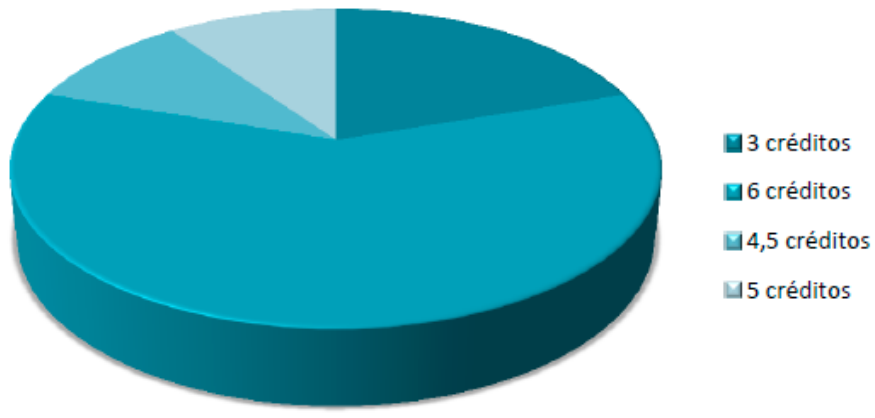

Estudio de las guías docentes de EA

En este apartado vamos a dar un paso más en el análisis de la ambientalización de los estudios de CA, apoyándonos para ello en la revisión de 19 guías docentes de las 20 Universidades en las que se imparte EA. La ausencia de una de las guías docentes se debe a que se encontraba en proceso de elaboración en el momento en que se efectuó la consulta. 
La revisión referida se ha abordado tomando como referencia externa una serie de indicadores de ambientalización (Tabla IV) extraídos a partir de los documentos emanados de las diferentes reuniones internacionales claves (Figura III) para la delimitación conceptual de la EA (véase, p. ej., Alonso, 2010). En dicha tabla se recogen los porcentajes de presencia de los anteriores indicadores en las guías docentes revisadas.
Tras analizar las competencias presentes en las guías docentes, se verifica la presencia de los ocho indicadores anteriormente comentados y el surgimiento de otros ocho nuevos con un carácter de competencias transversales, seguramente inspirados en la "filosofía” del Espacio Europeo de Educación Superior. En la Tabla IV se incluyen igualmente los nuevos indicadores identificados.

Figura III

Fuentes consultadas para elaborar los indicadores de ambientalización

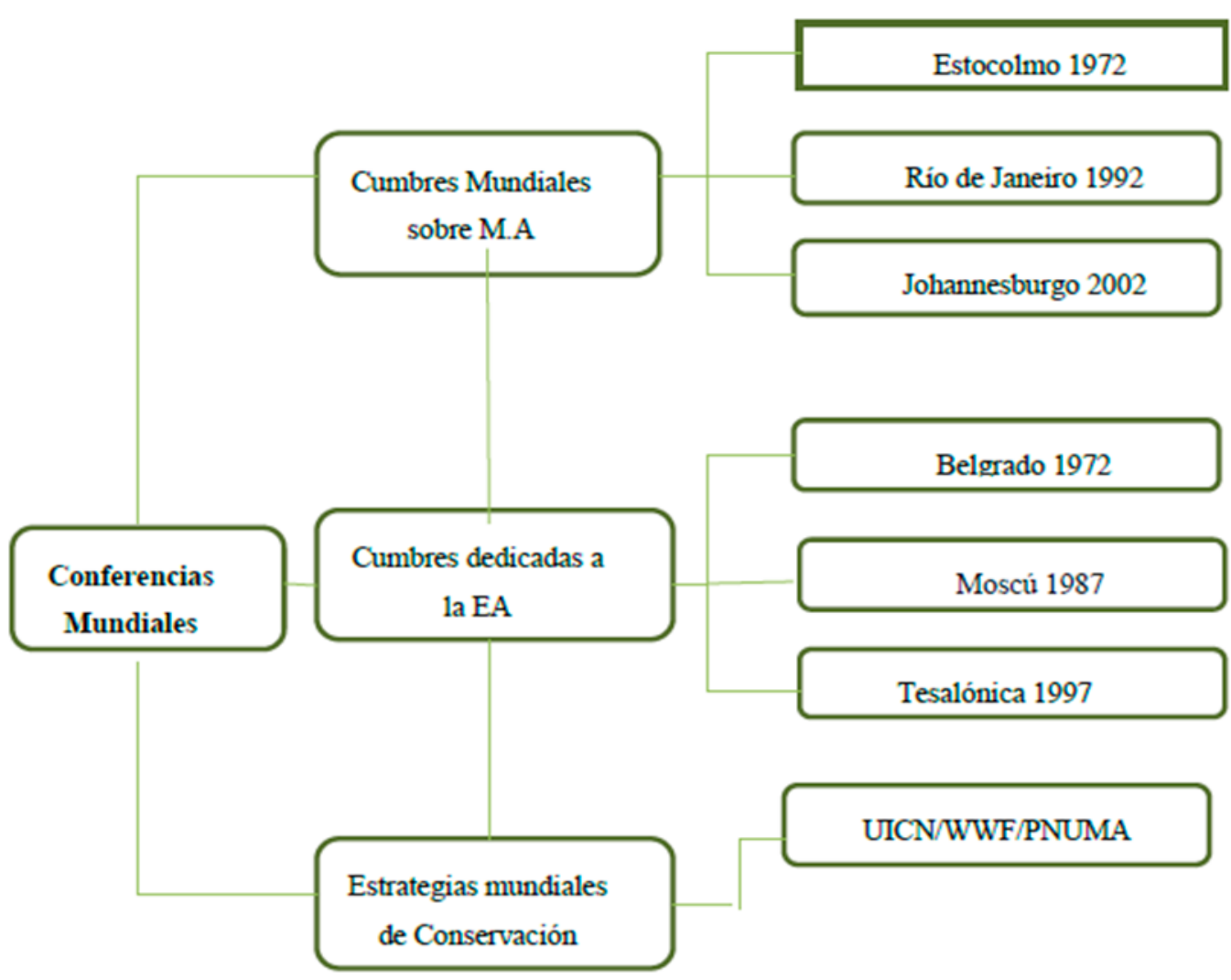




\section{Tabla IV}

Indicadores de ambientalización (1-8) considerados en el análisis de las guías docentes de EA y en las competencias explicitadas en aquellas

(9-16) (m.a.: medio ambiente) y porcentaje de presencia en aquellos

\begin{tabular}{|c|c|c|}
\hline Indicador $\mathbf{n}^{\mathbf{o}}$ & Descripción & Porcentaje \\
\hline 1 & Proceso permanente/continuo & $26 \%$ \\
\hline 2 & Interdisciplinario & $32 \%$ \\
\hline 3 & $\begin{array}{l}\text { Capacita para una convivencia armónica / interrelación m.a. y } \\
\text { ser humano }\end{array}$ & $63 \%$ \\
\hline 4 & Desarrolla valores y actitudes positivas frente al m.a. & $68 \%$ \\
\hline 5 & Aclara conceptos en materia de m.a. & $63 \%$ \\
\hline 6 & Desarrolla conciencia de los problemas m.a. existentes & $68 \%$ \\
\hline 7 & $\begin{array}{l}\text { Desarrolla hábitos y conductas para resolución y prevención de } \\
\text { problemas m.a. individuales y colectivos. }\end{array}$ & $84 \%$ \\
\hline 8 & $\begin{array}{l}\text { Contempla el desarrollo sostenible como un compromiso con las } \\
\text { generaciones futuras }\end{array}$ & $53 \%$ \\
\hline 9 & Capacidad de trabajo en equipo & $42 \%$ \\
\hline 10 & $\begin{array}{l}\text { Capacidad para comunicar ideas y argumentarlas, tanto a perso- } \\
\text { nas expertas como no expertas }\end{array}$ & $63 \%$ \\
\hline 11 & $\begin{array}{l}\text { Capacidad para hacer una lectura comprensiva, análisis y sín- } \\
\text { tesis }\end{array}$ & $37 \%$ \\
\hline 12 & Elaborar unidades didácticas, planes y programas de EA & $84 \%$ \\
\hline 13 & Saber usar los recursos para la educación & $63 \%$ \\
\hline 14 & Capacidad para interpretar datos y generar juicios & $58 \%$ \\
\hline 15 & Aplicar las TIC & $26 \%$ \\
\hline 16 & $\begin{array}{l}\text { Comprender el concepto de EA como respuesta a la problemática } \\
\text { ambiental }\end{array}$ & $42 \%$ \\
\hline
\end{tabular}

Considerando los datos anteriores globalmente a través de los dos indicadores más frecuentes, el 7 y el 12, se podría resumir la finalidad de la EA como la de desarrollar hábitos y conductas para resolver y prevenir problemas ambiéntales, además de saber elaborar unidades didácticas, planes y programas de EA. Parece evidente que se prima la dimensión actitudinal de la EA, así como la profesional en cuanto al aprendizaje estratégico para intervenir en futuros escenarios educativos, sean formales o no formales.

En la otra vertiente, si se identifican los dos indicadores menos presentes, 1 y 15 , se podría decir que la EA no se concibe con demasiada frecuencia como 
un proceso continuo y permanente, y que la aplicación de las TIC aún no está presente en la mayoría de las guías docentes, a pesar de las posibilidades que aquellas albergan (Ojeda, Gutiérrez y Perales, 2011).

Si atendemos ahora a las orientaciones metodológicas presentes en las guías docentes, hemos adaptado como indicadores de buenas prácticas en EA los contemplados por García y Galiano (2003) (Tabla V). En esta Tabla repre- sentamos el porcentaje de alusiones que a los mismos se hace en las guías docentes analizadas.

Como puede apreciarse, existe una presencia relevante de todos los indicadores seleccionados, destacando el papel del profesor como guía y mediador en el proceso de enseñanza, así como el favorecimiento del aprendizaje en grupo y de la enseñanza en grupo, lo que resulta consustancial con los principios metodológicos aceptados para la EA.

\section{Tabla V \\ Indicadores metodológicos identificados en el análisis de las guías docentes de EA y porcentaje de presencia en aquellas}

(Fuente: adaptado de García y Galiano, 2003)

\begin{tabular}{|l|c|}
\hline \multicolumn{1}{|c|}{ Principio Metodológico } & $\begin{array}{c}\text { Porcentaje de aparición } \\
\text { en las guías docentes }\end{array}$ \\
\hline 1. Adoptar un enfoque globalizador de los contenidos & $50 \%$ \\
\hline 2. El profesor actuará como guía y mediador & $100 \%$ \\
\hline 3. Promover la motivación de los alumnos & $55 \%$ \\
\hline 4. Necesidad de garantizar aprendizajes funcionales & $65 \%$ \\
\hline 5. Favorecer el aprendizaje en grupo & $85 \%$ \\
\hline 6. La enseñanza será activa & $80 \%$ \\
\hline 7. Adoptar una adecuada selección y secuenciación de los contenidos & $75 \%$ \\
\hline 8. Adecuar la utilización de diferentes recursos & $60 \%$ \\
\hline
\end{tabular}

\section{La opinión de los estudiantes}

Finalmente, quisimos tomar en consideración la opinión de los propios estudiantes de CA mediante una encuesta de preguntas de opción múltiple (escala tipo Likert) con cuatro opciones de respuesta y otras de carácter abierto, relacionadas con la presencia de la EA en el grado de CA, con sus intereses laborales y con la concepción de EA que poseen (utilizando los indicadores presentes en las Tablas IV). En la Tabla VI se muestran los resultados de las preguntas de índole cuantitativa y en la Tabla VII los resultados de los dos ítems cualitativos analizados por palabras clave. Hay que tener en cuenta que dichas palabras podían estar presentes en respuestas múltiples de los estudiantes, por lo que los porcentajes totales suman más del $100 \%$. 


\section{Tabla VI}

Porcentajes de respuesta a las cuestiones cualitativas incluidas en la encuesta (MDes: muy en desacuerdo; Des: en desacuerdo; DeA: de acuerdo; MDa: muy de acuerdo; Ob: obligatoria; Op: optativa; LC: libre configuración)

\begin{tabular}{|c|c|c|c|c|}
\hline \multirow{2}{*}{ Cuestión } & \multicolumn{4}{|c|}{ Porcentajes de respuesta } \\
\hline & \multicolumn{2}{|c|}{ Sí } & \multicolumn{2}{|c|}{ No } \\
\hline $\begin{array}{l}\text { 1. ¿Tiene usted constancia de que se oferte la asignatura EA en } \\
\text { su título de Grado en CA? }\end{array}$ & \multicolumn{2}{|c|}{21} & \multicolumn{2}{|c|}{79} \\
\hline 2. Si es así, ¿estaría interesado en matricularse en ella? & \multicolumn{2}{|c|}{90} & \multicolumn{2}{|c|}{10} \\
\hline \multirow{2}{*}{$\begin{array}{l}\text { 4. En relación a la siguiente afirmación "la EA debería incluirse } \\
\text { en el plan de estudios del ambientólogo", usted está }\end{array}$} & MDes & Des & DeA & MDa \\
\hline & 3 & 0 & 55 & 42 \\
\hline \multirow{2}{*}{$\begin{array}{l}\text { 5. En caso de estar de acuerdo con la pregunta anterior, ¿Cuál } \\
\text { cree usted que debería de ser la modalidad de esta asignatura?: }\end{array}$} & $\mathbf{O b}$ & \multicolumn{2}{|c|}{ Opt } & $\mathbf{L C}$ \\
\hline & 41 & \multicolumn{2}{|c|}{48} & 11 \\
\hline \multirow{2}{*}{$\begin{array}{l}\text { 6. ¿En qué curso considera usted que debería impartirse la asig- } \\
\text { natura? }\end{array}$} & \multicolumn{2}{|c|}{ Ciclo $1^{\circ}$} & \multicolumn{2}{|c|}{ Ciclo $2^{\circ}$} \\
\hline & \multicolumn{2}{|c|}{49} & \multicolumn{2}{|c|}{51} \\
\hline \multirow{2}{*}{$\begin{array}{l}\text { 7. Para usted las previsiones laborales de este Grado a la hora de } \\
\text { matricularse fueron decisivas. }\end{array}$} & MDes & Des & DeA & MDa \\
\hline & 14 & 48 & 31 & 7 \\
\hline 8. La EA es una posible alternativa laboral de futuro & 0 & 14 & 55 & 31 \\
\hline 10.1. La EA es competencia del ambientólogo & 3 & 3 & 38 & 56 \\
\hline 10.2. La EA debe ser un proceso permanente & 0 & 0 & 45 & 55 \\
\hline 10.3. La EA debe ser un proceso multidisciplinar & 0 & 3 & 34 & 63 \\
\hline $\begin{array}{l}\text { 10.4. La EA debe capacitar para la convivencia armónica / inte- } \\
\text { rrelación medio ambiente y sociedad: }\end{array}$ & 0 & 0 & 38 & 62 \\
\hline $\begin{array}{l}\text { 10.5. La EA debe desarrollar valores y actitudes positivas frente } \\
\text { al medio ambiente (ética ambiental) }\end{array}$ & 0 & 0 & 24 & 76 \\
\hline $\begin{array}{l}\text { 10.6. La EA debe aclarar conceptos en materia de medio ambien- } \\
\text { te (ser capaces de aplicar la teoría a la } \\
\text { práctica) }\end{array}$ & 0 & 3 & 34 & 63 \\
\hline $\begin{array}{l}\text { 10.7. La EA debe desarrollar conciencia de los problemas } \\
\text { ambientales (identificación) }\end{array}$ & 3 & 0 & 24 & 73 \\
\hline $\begin{array}{l}\text { 10.8. La EA debe desarrollar hábitos y conductas para resolver y } \\
\text { prevenir problemas ambientales }\end{array}$ & 3 & 0 & 34 & 63 \\
\hline $\begin{array}{l}\text { 10.9. La EA debe incluir el Desarrollo Sostenible para no compro- } \\
\text { meter a las generaciones futuras }\end{array}$ & 10 & 0 & 45 & 45 \\
\hline
\end{tabular}




\begin{tabular}{|c|c|c|c|c|}
\hline $\begin{array}{l}\text { 10.10. La EA debe ayudar a obtener capacidad de trabajo en } \\
\text { equipo }\end{array}$ & 0 & 21 & 48 & 31 \\
\hline $\begin{array}{l}\text { 10.11. La EA debe ayudar a desarrollar la Capacidad para } \\
\text { comunicar ideas y argumentarlas, tanto a personas expertas } \\
\text { como no expertas }\end{array}$ & 0 & 10 & 48 & 42 \\
\hline $\begin{array}{l}\text { 10.12. La EA debe ayudar a desarrollar la capacidad para hacer } \\
\text { una lectura comprensiva, análisis y síntesis }\end{array}$ & 0 & 21 & 52 & 27 \\
\hline $\begin{array}{l}\text { 10.13. La EA debe ayudar a elaborar unidades didácticas, planes } \\
\text { y programas de EA }\end{array}$ & 0 & 0 & 62 & 38 \\
\hline 10.14. La EA debe ayudar a saber usar los recursos educativos & 0 & 7 & 48 & 45 \\
\hline $\begin{array}{l}\text { 10.15. La EA debe ayudar a desarrollar la capacidad para inter- } \\
\text { pretar datos y generar juicios }\end{array}$ & 0 & 10 & 55 & 35 \\
\hline 10.16. La EA debe aplicar las TIC & 3 & 7 & 48 & 42 \\
\hline $\begin{array}{l}\text { 10.17. La EA debe entenderse como una respuesta a la problemá- } \\
\text { tica ambiental. }\end{array}$ & 0 & 17 & 45 & 38 \\
\hline
\end{tabular}

\section{Tabla VII}

\section{Porcentajes de respuestas a las cuestiones cualitativas incluidas en la encuesta (m.a.: medio ambiente)}

\begin{tabular}{|l|l|c|}
\hline \multicolumn{1}{|c|}{ Cuestión } & \multicolumn{1}{|c|}{ Palabra clave } & $\begin{array}{c}\text { Porcentajes de } \\
\text { respuestas }\end{array}$ \\
\hline & Concienciar & 55 \\
& Adquirir conocimientos de m.a. & 45 \\
& Comprender el m.a. & 34 \\
3. ¿Qué es para usted la EA? & Plantearse el futuro & 31 \\
& Reducción del consumo y la contaminación & 31 \\
& Adquirir valores ambientales & 14 \\
& No contestan & 10 \\
\hline & Concienciar & 55 \\
9. ¿Cuál considera usted que & Formar profesionales que enseñen & 38 \\
debería ser el objetivo princi- & Comunicar & 31 \\
pal de la EA? & Actuar & 24 \\
& Adquirir valores & 24 \\
& Plantearse el futuro & 17 \\
\hline
\end{tabular}

Analizados estos datos globalmente nos permiten afirmar que:

- Cuestión 1. Existe un gran desconocimiento sobre la presencia de la EA en el grado que están estudiando pues, a pesar de figurar en plan de estudios, los estudiantes no podían matricularse ese año por implicar contratación de nuevo profesorado en los departamentos responsables de esta materia (Didáctica de las 
Ciencias Experimentales y Métodos de Investigación y Diagnóstico en Educación).

- Cuestiones 2 y 4. Por el contrario, el interés por ella es muy elevado.

- Cuestión 5. La modalidad de oferta que se prefiere se distribuye casi a partes iguales entre la optatividad y la obligatoriedad.

- Cuestión 6. Lo mismo sucede entre la opción de ofertarla en los primeros cursos o en los últimos del grado.

- Cuestión 7. La gran mayoría de los estudiantes tuvieron en cuenta las perspectivas laborales en la elección del grado. Esto reforzaría la idea de consolidar la EA dentro de las posibles salidas profesionales.

- Cuestión 3. En cuanto a la naturaleza de la EA, las respuestas que se identifican más con el ámbito actitudinal del aprendizaje (conciencia, valores, futuro, comportamientos) superan ligeramente a la vertiente más de conocimientos ambientales. Estos resultados difieren de los obtenidos para futuros maestros o para los recién licenciados, donde la tendencia parece ser inversa (Moreno y García, 2008; Cebrián y Junjent, 2014).

- Cuestión 9. En esta cuestión se refuerzan los resultados de la cuestión anterior, apareciendo dos nuevas palabras clave con una presencia significativa, la de "Formar profesionales" y la de "Comunicar". Sin lugar a dudas, dos de los grandes retos de la EA.

- Cuestiones 10.1-10.17. Las respuestas de los estudiantes a los indi- cadores extraídos de la literatura educativa y de las competencias contempladas en las guías docentes manifiestan un gran acuerdo con los indicadores de EA expuestos (IV). Sin embargo, aparece menos unanimidad en las cuestiones menos específicas del campo conceptual de la EA (cuestiones 10.10, 10.11, 10.12 y 10.15), consideradas como competencias transversales al título del grado (Tabla IV). No responde a esta tendencia general la cuestión 10.17, referida a la EA como "respuesta a la problemática ambiental", lo que llama la atención como un contrasentido puesto que se supone que la EA constituye una alternativa viable para cambiar el estado de cosas actual.

\section{Reflexiones finales}

A pesar de las limitaciones de este estudio, especialmente en cuanto a la extracción de la muestra de estudiantes participante, estimamos que a través de estas páginas ha quedado patente la significativa y creciente, aunque claramente insuficiente, presencia de la EA en los estudios de grado en CA. Dicha presencia se manifiesta en la interdisciplinariedad que la caracteriza desde el punto de vista de los departamentos universitarios responsables y las áreas de conocimiento a las que corresponden, desde la diversidad de contenidos contemplados, así como desde la pluralidad metodológica que orienta su enseñanza, tal como quedan reflejados en las 
guías docentes correspondientes; ello es consistente con la naturaleza holística de la EA. A pesar de que se sabe y se asume la importancia de la EA en las universidades españolas que ofertan el grado de CA, ya que en prácticamente todas ellas se incluye como una posible alternativa laboral la EA, sin embargo no en todos los casos se contempla al menos una asignatura en relación con la EA en los planes de estudios. Seguramente ello tenga que ver con las relaciones de poder internas en las propias universidades a la hora de elaborar los planes de estudio. Como reto quedaría la evaluación desde dentro de la implementación de las diferentes materias de EA, analizando las tareas realizadas (Morales et al., 2014; Cardeñoso et al., 2015) o las opiniones de los graduados y otros estamentos implicados (Hansmann, 2009).

A pesar de todas las recomendaciones nacionales e internacionales que apuntan a la necesidad de promover la EA en los niveles educativos formales y no formales, la realidad es que la oferta formativa reglada en la universidad española es ciertamente escasa, careciéndose por ello de un perfil o itinerario profesional definido y socialmente consolidado. No deberíamos ignorar la creciente presencia, más o menos explícita, de la EA en campañas de sensibilización, equipamientos ambientales, exposiciones, publicidad, medios de comunicación... y que requeriría la participación de profesionales cualificados para su diseño o interpretación.
Los resultados obtenidos en este análisis exploratorio, unidos a la opinión favorable hacia la EA manifestada por los estudiantes encuestados, podrían representar a nuestro juicio un punto de partida donde apoyarse los futuros ambientólogos para emprender el urgente y necesario proceso de profesionalización de la EA, a pesar de las dificultades de su puesta en marcha como materia curricular (Van Petegem, 2005). Ello no impide que otros profesionales de la educación formal y no formal asuman igualmente ese papel en los niveles obligatorios de la enseñanza, para lo cual sus correspondientes grados y posgrados de formación inicial debieran contemplar una formación básica y eficiente en EA (Gutiérrez y Perales, 2012; Sureda et al., 2014), condición necesaria si se pretende que en el futuro tales profesionales puedan incorporar los principios y métodos de la EA a su docencia habitual. Estas recomendaciones, junto con otras para los distintos niveles educativos, ya fueron formuladas en el año 1999 en el "Libro de la Educación Ambiental en España" (MMA, 1999), aunque muchas de ellas siguen pendientes hoy día de su concreción práctica.

La tendencia de completar la formación de los grados con un máster profesionalizador o de investigación puede colaborar en dar respuesta a esta demanda. A ello responde, por ejemplo, la experiencia del Máster interuniversitario en Educador/a Ambiental ${ }^{3}$.

Nuestra investigación evidencia solo una pequeña parte del problema, 
es decir, la disonancia entre la demanda social de impregnar la educación de conocimientos y actitudes ambientales, y la tímida respuesta institucional. Los principios de la EA debieran arraigar en la ciudadanía desde las edades más tempranas mediante su presencia en la educación formal y a través de experiencias bien diseñadas en la no formal, fomentando la continuidad en la adquisición de hábitos y conductas proambientales en la madurez, acompañada de eficaces campañas de comunicación ambiental por parte, esencialmente, de las instituciones y de las organizaciones ecologistas.

\section{Bibliografía}

ALONSO, B. (2010). Historia de la Educación Ambiental. Asociación Española de Educación Ambiental. Recuperado de http://goo.gl/gv3DNG [28 de mayo de 2015].

ATHMAN, J.A. y MONROE, M.C. (2001). Elements of Effective Environmental Education Programs. ERIC No: ED463936. Recuperado de http://eric. ed.gov/?id=ED463936 [13 de febrero de 2016].

CARDEÑOSO, J.M.; CUESTA, J. y AZCÁRATE, P. (2015). Un instrumento para analizar las actividades prácticas en la formación inicial del profesorado de Secundaria de Ciencias y Matemáticas desde la perspectiva de la sostenibilidad. Revista
Eureka sobre Enseñanza y Divulgación de las Ciencias, 12, n¹, 109-129. DOI: 10498/16927

CARLETON-HUG, A. y HUG, J.W. (2010). Challenges and opportunities for evaluating environmental education programs. Evaluation and Program Planning, $\mathrm{n}^{\circ} 33,159-164$. DOI: 10.1016/j.evalprogplan.2009.07.005

CEBRIÁN, G. y JUNYENT, M. (2014). Competencias profesionales en Educación para la Sostenibilidad: un estudio exploratorio de la visión de futuros maestros. Enseñanza de las Ciencias, 32, $\mathrm{n}^{\circ} 1,29-49$. DOI: http://dx.doi. org/10.5565/rev/ensciencias. 877

CIS (Centro de Investigaciones Sociológicas) (2007). Ecología y Medio Ambiente (III). Recuperado de http:// www.cis.es/cis/opencm/ES/1_encuestas/estudios/ver.jsp?estudio=8160 [12 de mayo de 2015].

CE (European Comission) (2014).Attitudes of european citizens towards the environment. Recuperado de http:// ec.europa.eu/public_opinion/archives/ebs/ebs_416_en.pdf [3 de junio de 2015].

FUNDACIÓN BIODIVERSIDAD (2010). Informe Empleo verde en una economía sostenible. Recuperado de http://goo.gl/PyGZKv [28 de abril de 2015].

GARCÍA, J.E. (2004). Educación Ambiental, constructivismo y complejidad. Sevilla: Ed. Díada.

\footnotetext{
${ }^{3}$ https://www.uco.es/estudios/idep/masteres/educador-ambiental.
} 
GARCÍA, C. y GALIANO, M. (2003). Métodos y estrategias para la Educación ambiental. Eúphoros, $\mathrm{n}^{\circ} 6$, 249-260. Recuperado de https://goo. gl/J3quzf [11 de febrero de 2016]

GIL, E. (2004). La percepción de los problemas ambientales en Andalucía. Granada: Consejería de Medio Ambiente de la Junta de Andalucía.

GUTIÉRREZ, J. y PERALES, F.J. (2012). Ambientalización curricular y sostenibilidad. Nuevos retos de profesionalización docente. Profesorado, 16, $\mathrm{n}^{\circ} 2,5-14$.

HANSMANN, R. (2009). Linking the Components of a University Program to the Qualification Profile of Graduates: The Case of a Sustainability-Oriented Environmental Science Curriculum. Journal of Research in Science Teaching, $\mathrm{n}^{\circ} 46,537-569$. DOI 10.1002/tea.20286

MEDRANO, F. (1997). Evaluación de los programas de Educación Ambiental. En R. MENDOZA CASTELLÓN (coord.), Actas de la VIII aula de ecología educación ambiental (103-111). Recuperado de http://goo.gl/vjFyYj [10 de febrero de 2016].

MMA (Ministerio de Medio Ambiente (2009). Libro Blanco sobre la Educación Ambiental en España. Recuperado de http://goo.gl/P9w3Ij [15 de junio de 2015].

MORALES, A.J.; CAURÍN, C.; SENDRA, C. y PARRA, D. (2014). Aprendiendo a plantear problemas en el medio. Análisis de una experiencia con estudiantes del Máster de Investigación en Didácticas específicas. Didáctica de las Ciencias Experimentales y Sociales, $\mathrm{n}^{\circ} 28,65-81$. DOI: $10.7203 /$ DCES.28.3848

MORENO, E. y GARCÍA, J. (2008). La educación ambiental y el desarrollo sostenible: conceptualización del profesorado de formación inicial de secundaria. Didáctica de las Ciencias Experimentales y Sociales, ${ }^{\circ}$ 22, 203-218.

OJEDA, F.; GUTIÉRREZ, J. y PERALES, F.J. (2011). TIC y Sostenibilidad: Obstáculos y Posibilidades Para los Educadores Ambientales. Profesorado, 15, $\mathrm{n}^{\circ} 11,263-313$.

PERALES, F.J. (2006). Percepción ambiental en futuros maestros de Educación Primaria. Comunicación a los XXII Encuentros de Didáctica de las Ciencias Experimentales. Zaragoza.

PERALES, F.J. (2010a). Retos y dificultades para una educación ambiental informal.Alambique. Didáctica de las Ciencias Experimentales, n64, 23-35.

PERALES, F.J. (2010b). Cambios en la percepción ambiental de los futuros maestros de educación primaria. Comunicación al II Congreso Internacional de Didácticas. Gerona.

PONCE, I. y TÓJAR, J.C. (2014). Análisis de competencias y oportunidades de empleo en una enseñanza de posgrado. Propuesta metodológica de evaluación en un máster interuniversitario de educación ambiental. Profesorado, 18, $\mathrm{n}^{0} 2,171-187$. Recuperado de http://www.ugr.es/local/recfpro/ rev182COL2.pdf 
SUREDA-NEGRE, J.; OLIVER-TROBAT, M.; CATALÁN-FERNÁNDEZ, A. y COMAS-FORGAS, R. (2014). Environmental education for sustainability in the curriculum of primary teacher training in Spain. International Research in Geographical and Environmental Education, 23, ${ }^{\circ} 4$, 281-293. DOI: 10.1080/10382046.2014.946322

TÁBARA, J.D. (2001). La medida de la percepción social del Medio Ambiente. Una revisión de las aportaciones realizadas por la sociología. Revista Internacional de Sociología, $\mathrm{n}^{\circ} 28$, 127-171.

UICN-UNEP-WWF (1970). Carta de Nevada. Nevada, E.U: UICN.

VAN PETEGEM, P.; BLIECK, A.; IMBRECHT, I. y VAN HOUT, T. (2005). Implementing environmental education in pre-service teacher training. Environmental Education Research, 11(2), 161-171. DOI: 10.1080/1350462042000338333 
